\title{
PROPOSIÇÃO DE UMA SISTEMÁTICA DE AVALIAÇÃO DE APRENDIZAGEM NA FORMAÇÃO DEE ADMINISTRADORES COM BASE NO PROCESSO GERENCIAL
}

\author{
PROPOSITION OF A SYSTEMATIC EVALUATION \\ OF LEARNING FOR MANAGERS FORMATION BASED \\ ON MANAGERIAL PROCESS
}

\author{
Recebido 10/07/2012 \\ Aceito 09/09/2012 \\ Daniel Nascimento e Silva ${ }^{1}$ \\ Chiara da Silva Simões ${ }^{2}$ \\ Márison Luiz Soares ${ }^{3}$ \\ Ana Maria Alves Pereira ${ }^{4}$
}

\section{RESUMO}

Os administradores são os resultados de sistemas de produção racionalmente desenhados. 0 administrador é o resultado de uma série de conhecimentos, habilidades e padrões de comportamento, cuja utilidade é diretamente proporcional à consistência de sua formação profissional, ou seja, àquele sistema de produção racionalmente desenhado. Assim, este artigo tem como objetivo apresentar uma sistemática de avaliação de aprendizagem baseada nas funções do processo gerencial: planejamento, organização, direção e controle. A sistemática consiste em dez etapas: definição dos conhecimentos a serem avaliados, definição das habilidades a serem avaliadas, escolha do formato do teste, redação das questões, definição das normas de resolução das questões, avaliação do pré-teste, realização do teste, organização dos dados, avaliação dos resultados, análise das causas de fracasso e análise do replanejamento. Os resultados mostram que o processo de formação de administradores pode ser otimizado se as instituições de ensino superior empregarem o sistema desenhado em conformidade com o perfil do egresso do curso.

Palavras-chave: Avaliação da aprendizagem, formação de administradores, formação profissional, processo gerencial.

\footnotetext{
${ }^{1}$ Possui graduação em Administração pela Universidade Federal do Pará - UFPA, mestrado em Administração e doutorado em Engenharia de Produção pela Universidade Federal de Santa Catarina - UFSC. Atualmente é professor do Instituto Federal de Educação, Ciência e Tecnologia do Amazonas. Manaus, Amazonas, Brasil. E-mail: danielnss@gmail.com.

${ }^{2}$ Possui graduação em Pedagogia pela Universidade Luterana do Brasil - ULBRA. E-mail: chiaraclara1@hotmail.com.

${ }^{3}$ Possui graduação em Administração pela Universidade do Vale do Itajaí - UNIVALI, mestrado e doutorado em Engenharia de Produção pela Universidade Federal de Santa Catarina - UFSC. Atualmente é professor adjunto da Universidade Federal do Espírito Santo - UFES. Vitória, Espírito Santo, Brasil. E-mail: marison@matrix.com.br.

${ }^{4}$ Possui graduação em Pedagogia, especialização em Engenharia da Produção e mestrado em Ciências Ambientais e Florestais pela Universidade Federal do Amazonas - UFAM. Atualmente é servidora pública federal da carreira do magistério do ensino básico, técnico e tecnológico. Manaus, Amazonas, Brasil. E-mail: anamaria.ifam@gmail.com.
} 


\begin{abstract}
Managers are the outcomes of production systems rationality designed. The manager as outcome is a mix of knowledge, skills and behavior pattern whose utility is directly proportional to the consistency of your professional formation, just the production systems rationality designed. Thus, this paper aims to present a systematic evaluation of learning based on managerial process functions: planning, organization, directing and control. It consist of ten steps: definition of knowledge to be evaluate; definition of skills to be evaluate; choosing of test framework; writing of questions; definition of test solution norms; evaluation pre-test; testing; organization of data; evaluation of results; and analyzing the causes of failure and replanning. The results show that manager formation process can be optimized if higher education institutions use the system in accordance with the course objectives.
\end{abstract}

Keywords: evaluation of learning, managers formation, professional formation, managerial process.

\title{
1 INTRODUÇÃO
}

O processo gerencial é o sequenciamento lógico de quatro etapas: planejamento, organização, direção e controle. Esse processo é o responsável pela concretização dos objetivos organizacionais em todos os níveis, estratégico, tático e operacional. Como cada disciplina componente da matriz curricular dos cursos de administração é focada no alcance de objetivos, a avaliação da consecução ou não desses objetivos pode ter, na aplicação do processo gerencial, uma importante ferramenta para a elevação da qualidade dos profissionais de gestão das instituições de ensino superior brasileira. Isso quer dizer que a aplicação do processo gerencial na formação de administradores pode proporcionar às instituições de ensino superior o mesmo grau de perfeição que as organizações que o aplicam adequadamente conseguem ao produzir seus produtos e serviços.

Entre os vários aspectos que podem ser imediatamente melhorados no processo de formação profissional, está a forma como é avaliada a aprendizagem desses profissionais. Na verdade, é difícil encontrar exemplos nacionais de sistemas de avaliação consistentes, lógicos e estruturalmente integrados com a cadeia de valor do curso. Ora, se um curso é uma organização e, como toda organização, produz alguma coisa, os egressos do curso representam seus produtos, e quem os emprega são os clientes do curso. Assim, um sistema de avaliação da aprendizagem deve estar de acordo com essa sistemática valorativa da cadeia ou do processo de agregar valor continuamente até que o profissional de administração apresente os conhecimentos, as habilidades e os comportamentos de administrador.

Nesse sentido, este artigo tem como objetivo demonstrar uma sistemática de avaliação da aprendizagem passível de ser aplicada para a melhoria da qualidade da formação do administrador. Para isso, concentra-se na função controle, quarta etapa do processo gerencial, e mostra como padrões podem ser gerados na avaliação, de que maneira o processo de mensuração pode ser integrado à avaliação e, caso haja necessidade de retificações, como se pode fazer a recuperação de aprendizagem que efetivamente leve ao aluno os conhecimentos, as habilidades e os comportamentos que este não obteve naturalmente ao longo das atividades de ensino regulares.

Este artigo está estruturado da seguinte forma: nesta introdução, sua primeira parte, é apresentado o objetivo do texto; em seguida, é delimitado o escopo teórico que fundamenta o objetivo do texto, o qual constitui a segunda parte; a terceira parte detalha o procedimento metodológico utilizado para gerar as descobertas da investigação; a quarta etapa apresenta e discute os resultados obtidos e, finalmente, a quinta etapa expõe a conclusão e a recomendação de estudos futuros. 


\section{REFERENCIAL TEÓRICO}

Administração é o processo de planejar, organizar, dirigir e controlar recursos para o alcance de objetivos organizacionais. Esse processo, dividido em quatro etapas, é também chamado de processo gerencial ou processo administrativo. A avaliação, por sua vez, é a terceira etapa da função controle, quarta etapa do processo gerencial. Isso significa que qualquer proposta de avaliação é inconsistente se as etapas anteriores não estiverem coerentemente determinadas. 0 que se pretende mostrar é que a avaliação faz parte de uma sistemática mais abrangente, chamada controle, que, por sua vez, faz parte de um mecanismo maior e mais complexo, intitulado Administração. Assim, todo sistema de avaliação tem de dar conta do microssistema gerencial, sob pena de se tornar inócuo ou ilógico.

Toda organização parece "condenada" à aprendizagem, toda organização aprende (CAVUS, 2010; KOC; ERGINBAS, 2011; CAVUS; MOMANI, 2009; CUPPEN, 2012). No entanto, esse processo de aprendizagem não procede de forma assistemática, desordenada. Como mostrou Lulsegged (1984), em um estudo datado da década de 1980, no chamado terceiro mundo daquela época, no qual o Brasil se inseria, a aprendizagem era feita de forma desordenada. Porém, atualmente, os recursos virtuais (WRZESIEN; RAYA, 2010) e reais são inúmeros e atuam no esforço concentrado de elevar a qualidade do profissional de administração que é inserido no mercado. Além disso, atuam em uma perspectiva multidisciplinar, a qual não emprega apenas esquemas tecnológicos, mas também recursos de organização do trabalho e da produção, que são criados constantemente neste sentido. É por essa perspectiva de esforço que a presente proposta de sistemática de avaliação da aprendizagem deve ser interpretada.

\subsection{A função planejamento}

O planejamento tem a finalidade de responder a duas questões essenciais, sem as quais se torna impossível a atividade gerencial: "o que fazer?" e "como fazer?". A resposta fornecida à primeira pergunta identificará os objetivos a serem alcançados; a resposta à segunda fornecerá as estratégias a serem utilizadas enquanto caminhos que levarão ao destino (objetivos) pretendido (MARQUEZ; GUPTA, 2006).

Em termos de planejamento da aprendizagem, o docente precisa saber com precisão aonde quer que o discente chegue. Em termos práticos, isso se faz escolhendo os conhecimentos, as habilidades e o padrão de comportamento que ele este deverá incorporar ao final de determinado horizonte de planejamento (MONTOYA-DEL-CORTE; FARÍAS-MARTÍNEZ; 2009; ALMEIDA; PERES, 2012). Conhecimentos são esquemas lógicos que organizam o raciocínio e dão sentido àquilo que se faz, na prática, ao aplicá-los; as habilidades são formas específicas ou gerais de aplicar esses esquemas lógicos e, por último, os padrões de comportamento são a materialização psíquica da aprendizagem adquirida, a qual modifica o comportamento daquele que aprendeu, isto é, quem aprendeu modifica o seu comportamento. 


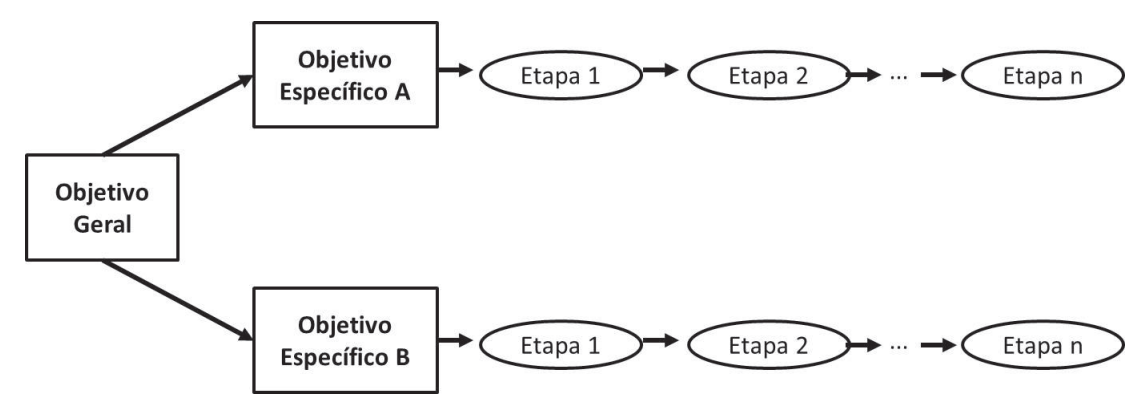

Figura 1. Exemplo de esquema lógico da função planejamento de aprendizagem.

Escolhidos os objetivos a serem alcançados, para completar a etapa de planejamento a etapa seguinte deve centrar-se no caminho a ser percorrido, ou seja, na estratégia que levará à sua consecução (JOHNSON; CLARK, BARCZAK, 2012; SING; WATSON; WATSON, 2002). A elaboração da estratégia leva em consideração esquemas lógicos criados pelo professor, mas perfeitamente diagramáveis em fluxogramas, como mostra a Figura 1, e a previsão do uso de recursos em cada etapa do esquema lógico criado. O esquema lógico é o desdobramento do objetivo geral pretendido em objetivos específicos, seguido da criação de outros esquemas lógicos para cada objetivo específico desdobrado do objetivo geral pretendido. Nenhum plano estará completo caso não apresente um caminho detalhado para cada objetivo específico pretendido.

A conjunção de objetivos e estratégias viáveis permite que, quando a última atividade da última etapa do esquema lógico for finalizada, cada objetivo específico seja alcançado e, por extensão, que o objetivo geral seja concretizado. Essa dinâmica será fundamental tanto para a compreensão da aplicação do processo gerencial na avaliação da aprendizagem quanto para a construção dos elementos de controle, especialmente a dos instrumentos de mensuração.

\subsection{A função organização}

A função organização preocupa-se em disponibilizar os recursos para que os objetivos previstos no plano possam ser alcançados (AMIT; BELCOURT, 1999; LONDON; SMITHER, 2002). Nenhum objetivo pode ser alcançado sem que consuma recursos. Dito de outra forma, todo objetivo consome recursos e, por isso, tem um custo, uma vez que todo recurso tem um preço. A questão básica que deve ser respondida aqui é a seguinte: "quais são os recursos necessários para executar as estratégias desenhadas para cada objetivo?". Em termos de atividades docentes, esses recursos configuram o que se pode chamar de recursos didáticos (tais como quadro negro e pincel atômico), infraestrutura física (laboratórios, veículos para transportes da turma etc.) e pessoal de apoio (laboratoristas, motoristas, dentre outros).

Evidentemente que, ainda que os alunos representem sempre a matéria-prima do processo de formação de administradores, podem desempenhar diversos papéis na função organização. O que importa, aqui, é que se compreenda que, para cada etapa da estratégia, recursos deverão ser consumidos e devem estar disponíveis na data prevista. Em disciplinas cujas aulas se concentram em salas fixas, geralmente a previsão de recursos é simplificada; noutras disciplinas, em que a aprendizagem ocorre em contextos ambientais, os recursos devem ser providenciados com bastante antecedência. Nota-se, aqui, naturalmente, a preocupação constante de que a estratégia seja implementada tal como foi desenhada para garantir a concretização dos objetivos pretendidos. 


\subsection{A função direção}

A função direção é centrada no elemento humano da administração, principal causa de insucessos de planos e, ao mesmo tempo, fator primordial da concretização dos objetivos organizacionais (AMIT; BELCOURT, 1999; BULLER; McEVOY, 2012). Do ponto de vista da aplicação do processo gerencial na formação de gestores, o docente, por meio da aplicação de ferramentas típicas de gestão de pessoas, poderá criar um mapa do perfil da turma, em que as características da personalidade individual de cada um sejam identificadas. Esse mapa servirá de referência para a criação dos esquemas de motivação, para evitar que uma parte do alunado transforme-se em indivíduos indiferentes ou, até mesmo, agressivos e adversários na implementação das estratégias de aprendizagem. $O$ sucesso da aprendizagem vai depender, em diversas situações, da habilidade do docente de manter o interesse do aluno nas questões de aprendizagem até que este, por si só, automotive-se, como propõem os estudos motivacionais (VARGAS; BIRRER; MINELLO, 2012; CORDEIRO; SILVA, 2012).

Como não existe esquema de motivação sem a contraparte da liderança, como afirmam Sweetser e kelleher (2011) e Li, Tan e Teo (2012), é necessário, também na aprendizagem de gestores, que o exercício de mando-obediência seja implementado. Esse exercício, nas atividades docentes, é passível de inúmeras "autodescobertas" por parte do alunado, como, por exemplo, o fato de que a "passividade ativa" é responsável pelo sucesso organizacional e o de que todo grupo tem um líder, ainda que os liderados não percebam. Assim, de forma latente ou explícita, o docente precisa valer-se dos esquemas de liderança para facilitar a implementação das estratégias.

Ficaria mais precisa a redação assim "A comunicação completa os desafios de se implementar a função direção. Estudos como os de Johansen, Aggerholm e Frandsen (2012) e Schwarz e Pforr (2011) demonstram as crises gerenciais que sistemas de comunicações inadequados podem gerar. Essas comunicações, são de cinco níveis: descendentes, representando os objetivos, as normas, as instruções e outros tipos de mensagens que partem da instituição e do docente para os discentes; ascendentes, que partem dos alunos para o docente e a instituição, principalmente em forma de demanda por orientações, saneamento de dúvidas e outros tipos; laterais, feitas entre os alunos de um mesmo grupo e entre alunos de outros grupos da mesma turma; diagonais, quando as comunicações partem e/ou envolvem alunos de diferentes grupos, turmas, docentes e pessoal da instituição e, por fim, ambientais, quando envolve comunicações da instituição com o ambiente externo. $O$ docente de gestão poderá, com facilidade, elaborar o quadro de comunicações que levem à condução, dentro de padrões de normalidade, de seu alunado ao aprendizado pretendido.

\subsection{A função controle}

Os esforços humanos são racionalizados para que o desempenho alcançado seja, pelo menos, razoável. Por isso, cada etapa precisa ser controlada, para que aumente a probabilidade de sucesso; esse é o desafio do controle e da mensuração de desempenho, como se pode verificar a partir dos estudos de Arend (2010) e Chen (2011). A função controle é a última etapa do processo gerencial e, também, a que dá início a um novo ciclo, o que demonstra que o processo gerencial é constante e perene. Toda forma de controle, para que possa ser viável e garantir que os objetivos possam ser alcançados, é implementada em quatro etapas. A primeira etapa é a identificação de padrões comparativos ou o estabelecimento de indicadores de desempenho. Por exemplo, para o objetivo específico de habilidade "ser capaz de redigir objetivos operacionais", o padrão poderia ser "capaz ou incapaz"; para o objetivo específico de conhecimento "compreen- 
der a importância do fluxo de caixa para o sucesso financeiro empresarial", o padrão poderia ser "compreende bem, compreende medianamente e não compreende". O esquema comparativo permite que o docente proceda de forma consistente na avaliação da aprendizagem e realize as outras etapas do processo de controle do alcance dos objetivos pretendidos.

A mensuração é a segunda etapa do processo de controle e tem a finalidade de registrar, quantitativamente, a execução das atividades que levarão à consecução de cada objetivo específico (BISBE; MALAGUEÑO, 2012). Por exemplo, para o objetivo específico "ser capaz de elaborar planos operacionais", se a estratégia prevê a realização de três provas, a cada prova seria gerado um escore (nota) que, por sua vez, iria compor a terceira etapa, que é a avaliação propriamente dita. O que se deve ter em mente, portanto, é que a mensuração representa pura e simplesmente a aquisição de um escore, enquanto dado, que será organizado na etapa seguinte. É recomendável que a mensuração seja feita em conformidade com as técnicas de escolha de indicadores operacionais de desempenho.

A terceira etapa da função controle é a avaliação. A avaliação funciona como uma forma de julgamento decorrente da comparação do resultado da mensuração com o padrão de controle (WU; LIN; CHANG, 2011; GUERRA-LÓPEZ; TOKER, 2012). Para que isso seja feito, é preciso que os escores obtidos ao longo do processo de mensuração sejam transformados a fim de se tornem passíveis de comparação. Por exemplo, se a nota média para aprovação em uma disciplina for 7,0, essa nota média é o padrão; em seguida procede-se ao processo de mensuração ao se realizar diferentes provas e trabalhos, nos quais é obtida uma nota ou um escore. Na avaliação, essas notas são convertidas em um único número sintetizador (geralmente a média aritmética), que será comparado com o número padrão: se o escore for igual ou superior ao padrão, o aluno é aprovado; se for inferior, será objeto de recuperação.

A quarta etapa da função controle é a retificação ou o replanejamento. No caso de avaliação de aprendizagem, à semelhança de qualquer outro processo de produção, a finalidade é identificar os motivos da não conformidade (estar fora do padrão, ou seja, ter obtido média inferior ao mínimo necessário) para que sejam eliminados e, com isso, a partir de uma nova avaliação, os discentes-alvo do replanejamento recoloquem-se no padrão.

\subsection{0 processo de avaliação de aprendizagem}

O processo de avaliação de aprendizagem para a formação de administradores está focado no construto "competência". A competência é o conjunto de conhecimentos, habilidades e comportamentos necessários para que o administrador possa desenvolver a contento as atribuições gerenciais. A competência essencial é aquela que fornece ao profissional ser mais competente dentre os competentes, como se depreende de Petts (1997) e Major, Asch e Cordey-hayes (2001). Dessa forma, a avaliação da aprendizagem, de forma específica, concentrar-se-á nessa tríade: conhecimentos, enquanto esquema lógico e interpretativo que permite ao profissional conhecer e compreender o universo organizacional; habilidades, como as técnicas, ferramentas, metodologias e todo o instrumental necessário que o gestor precisa saber criar/ter para executar as funções gerenciais e comportamentos gerenciais, que são a comprovação inconteste de que o profissional detém os conhecimentos e as habilidades gerenciais. Todo aprendizado, portanto, seja de conhecimentos e/ou habilidades implica, sempre, modificação de comportamento: se o discente não mudou o comportamento, não aprendeu.

Depois de executadas todas as atividades de ensino (em que conceitos, construtos, variáveis, dimensões e categorias analíticas foram apresentados, demonstrados, testados, enfim, con- 
solidaram-se enquanto aprendizagem) é chegada a etapa de avaliar em que grau se deu essa consolidação. Nas atividades de ensino é fundamental, portanto, que sejam esclarecidas as relações multidimensionais entre conhecimentos (esquemas lógicos) e seus desdobramentos em questões práticas, com a necessidade, inclusive, de demonstração de que técnicas, metodologias e todos os instrumentos gerenciais são decorrentes dos conhecimentos, estejam eles baseados não em teorias. O domínio pleno desse esquema lógico e seu consequente desdobramento em mudanças de comportamentos é o que confere a competência ao profissional. A competência é maior ou menor em conformidade com o grau de amplitude e profundidade de consolidação desse esquema.

A primeira etapa do processo de avaliação de aprendizagem é a definição de quais conhecimentos serão avaliados. Esses conhecimentos devem ser sintetizados para que não haja dúvidas para os avaliados e tampouco para o avaliador sobre qual a extensão e a profundidade da avaliação. Por exemplo, para avaliar o conhecimento "processo gerencial", o que o avaliador vai testar nas provas e outras atividades de que se utilizará para gerar os escores e/ou dados de mensuração deve estar claro para todos; o discente tem que demonstrar que compreende a lógica do processo gerencial. Assim, deduções como "não há a possibilidade de execução da função organização se a função planejamento não estiver finalizada" são consequências naturais de se compreender o básico sobre o "processo gerencial".

A segunda etapa é a definição de quais habilidades serão avaliadas. Nessa etapa, deve estar claro para o avaliador que a habilidade é a busca pelo que o aluno sabe fazer com aquilo que supostamente aprendeu. Afinal, aquele que sabe, deve ter capacidade para demonstrar e fazer. Enquanto profissional, todo administrador precisa ter inúmeras habilidades decorrentes dos conhecimentos que aprendeu. Por exemplo, se aprendeu a função planejamento do "processo gerencial", deve saber redigir objetivos gerais e específicos, elaborar estratégias, relacionar os objetivos específicos com o objetivo geral, integrar estratégias com objetivos etc. Cada uma dessas habilidades pode ser testada. O resultado do teste é um número, escore ou dado que serão considerados no processo de avaliação.

A terceira etapa foca os instrumentos de teste, os quais são inúmeros. Contudo aqui será considerado apenas o instrumento mais utilizado, que é a prova bimestral, de maneira que esta sistemática seja plenamente compreendida. $O$ teste desta sistemática é composto por dois tipos de questões, à semelhança do Exame Nacional de Desempenho de Estudantes (Enade), objetivas e discursivas. Nas questões objetivas será testada a aprendizagem dos conhecimentos e das habilidades isoladamente; nas discursivas, por meio de respostas escritas, conhecimentos e habilidades serão testados em conjunto. As questões objetivas serão sempre em número par, em consonância com a seguinte regra: cada conhecimento testado gera uma habilidade a ser testada. Por exemplo, o conhecimento das regras para fazer plano orçamentário será testado tanto "teoricamente" quanto "praticamente" nas questões objetivas. Isso resulta, sempre, em teste do conhecimento por meio de uma questão específica para tal e reteste deste conhecimento através da habilidade do aluno de aplicá-lo, também através de outra questão específica para isso.

A quarta etapa é a redação das questões. As questões devem ser redigidas em conformidade com o grau de profundidade e amplitude que a avaliação requer. Devem começar com um contexto sintetizado, em seguida, os dados e/ou informações necessárias para o raciocínio devem ser apresentadas e finalizadas, apresentando-se o problema que deve ser solucionado. Finalizam-se as questões objetivas com as alternativas, e as discursivas com as orientações para a resposta. No caso das questões discursivas, o avaliador deve elaborar um padrão de resposta, que pode ser uma pequena lista de assuntos que, obrigatoriamente, o discente deve citar explicitamente na redação de sua resposta. A contemplação dos itens desta lista conferirá a nota, desde que o formato de redação seja obedecido. 
A quinta etapa é a redação das normas de resolução do teste. Aqui devem ser esclarecidas questões como: a) tempo médio para a resolução de cada questão objetiva, b) tempo médio para a resolução de cada questão discursiva, c) formato da redação (mínimo e máximo de linhas, número de parágrafos, dentre outras especificações), d) material e instrumentos necessários para a resolução das questões e outras questões relevantes.

A sexta etapa é o pré-teste da turma. Essa etapa é extremamente importante para que a turma tenha uma noção precisa de como é o teste, conheça as regras, mensure o tempo, saiba o grau de amplitude e dificuldade das questões, dentre outros aspectos relevantes. É nessa etapa que o discente saberá como se preparar para o teste real.

A sétima etapa é a aplicação do teste. Apesar de essa etapa referir-se apenas à sistemática do processo de avaliação, é sempre de importante o esclarecimento de que pelo menos dois testes precisam ser aplicados para aferir com alto grau de probabilidade de acerto o real estado de aprendizagem de cada aluno e, por extensão, da turma. Esta etapa é a primeira etapa de mensuração.

A oitava etapa é a organização dos dados e consiste na tabulação, questão por questão, aluno a aluno, dos erros e acertos verificados no teste. Como será mostrado no exemplo demonstrativo mais à frente neste artigo, nessa etapa pode-se utilizar qualquer planilha eletrônica que contenha a possibilidade de cálculos simples, como moda, média, desvio padrão, correlação e outras estatísticas simples. Essa etapa envolve o final da mensuração e o início da etapa de avaliação, na lógica da função controle do processo gerencial.

A nona etapa é a avaliação propriamente dita. Aqui os escores individuais do teste são comparados com o padrão e, imediatamente, gera-se a avaliação, o julgamento sobre se o aluno aprendeu ou não. Essa sistemática permite ver a realidade da aprendizagem do aluno e da turma com profundidade, ou seja, mostra que conhecimentos e habilidades faltam a cada aluno e para a turma toda. O que importa, aqui, não é apenas quantos alunos foram aprovados, mas, essencialmente, quantos não tiveram sucesso.

A décima etapa consiste na análise das causas de fracasso dos alunos e no replanejamento do objetivo a ser alcançado e da estratégia a ser seguida. Através de algum instrumento de análise de defeitos ou de problemas, como o diagrama de Ishikawa, podem-se detectar com precisão as causas do fracasso de qualquer aluno. Com o diagnóstico feito, podem-se criar estratégias para corrigir o problema, eliminar as causas do fracasso; assim, o processo recomeça.

\section{METODOLOGIA}

Para a realização deste estudo demonstrativo, a unidade de análise foi constituída pelos alunos da disciplina Gestão e Empreendedorismo do curso de graduação de uma Instituição de Ensino Superior de Manaus, capital do Estado do Amazonas. O nível de análise é organizacional, uma vez que a turma toda foi tomada como uma organização: tem mais de um indivíduo com objetivos em comum (WALTER; BRUCH, 2010). A perspectiva de análise é sincrônica ou transversal, uma vez que os resultados aqui obtidos representam uma explicação estática, parada no tempo, como se fosse uma fotografia do fenômeno "aprendizagem" no exato momento em que se procedeu a avaliação dos alunos da turma.

O tipo de estudo é descritivo, em consonância com o objetivo do estudo, que é a descrição do funcionamento da sistemática de avaliação, e com o estoque de conhecimentos sobre o fenômeno "avaliação da aprendizagem" (ROBSON et al., 2012), que permite a formulação de relação entre variáveis já testadas em outros estudos, tais como conhecimentos, habilidades e competên- 
cias. As variáveis-objeto deste estudo foram quantificadas em torno de escores, em conformidade com o quadro teórico de referência, para que se pudesse demonstrar esta sistemática de avaliação, o que lhe confere o enquadramento no tipo de pesquisa quantitativa (USUNIER, 2011).

Os dados são todos de tipo primário, coletados por meio do teste de avaliação de aprendizagem, criado com base nas etapas descritas no marco teórico deste estudo. $O$ teste foi composto por 14 questões objetivas, das quais sete mediam conhecimentos e sete mediam habilidades. Duas questões discursivas completaram a avaliação. Os resultados foram organizados com o apoio de planilhas do Excel, a partir da qual foram gerados os resultados do desempenho de cada aluno e da turma, questão a questão. As estatísticas produzidas foram frequências simples, frequência percentual, média e desvio padrão.

As frequências simples foram utilizadas para gerar o escore de cada aluno e para indicar a quantidade de acertos da turma para cada questão; a frequência percentual serviu para indicar o desempenho da turma para cada questão; a média foi utilizada para saber o desempenho da turma em todas as questões objetivas, nas questões discursivas e gerar o desempenho global da turma e o desvio padrão foi usado para medir a distância de cada aluno em relação à média da turma. Os resultados foram expostos em um quadro sintetizador da avaliação.

A turma foi escolhida por conveniência: como um dos pesquisadores é docente da instituição e da turma, este combinou com a turma a realização do teste do sistema de avaliação e obteve $o$ aceite. Nessa turma predominam alunos jovens, com média de idade de 24 anos, limite superior de idade de 54 e inferior de 18, com classe modal 19 e desvio padrão de 5,4 anos. Nenhum deles tinha tido contato com esta sistemática de avaliação.

\section{RESULTADOS E DISCUSSÃO DA DEMONSTRAÇÃO DA SISTEMÁTICA}

O plano da disciplina prevê três blocos de conhecimentos específicos que devem ser atingidos ao final da disciplina; os alunos deverão ser capazes de: planejar e executar o processo gerencial, analisar organizações e suas relações com o ambiente externo e elaborar planos de negócios. Para efeito de teste dessa sistemática, escolheu-se uma parte do bloco "planejar e executar o processo gerencial", representado, neste artigo, pelo objetivo específico "elaborar e executar planos operacionais". Portanto, este será o objetivo do teste: saber se os alunos sabem ou não planejar e executar planos operacionais.

\subsection{Primeira etapa: definição dos conhecimentos a serem avaliados}

Para o desenvolvimento do teste, foram definidos os seguintes conhecimentos necessários, relativos à função planejamento: a) o que é planejamento, b) o que é objetivo, c) o que é estratégia, d) quais os elementos obrigatórios em um objetivo, e) quais os elementos obrigatórios em uma estratégia, f) qual a relação entre objetivo geral e objetivos específicos e g) qual a relação entre objetivos e estratégias.

\subsection{Segunda etapa: definição das habilidades a serem avaliadas}

As habilidades correspondentes aos conhecimentos que foram definidos para fazer parte da avaliação foram: a) como se planeja, b) como identificar um objetivo, c) como identificar 
uma estratégia, d) como se redige um objetivo, e) como se desenha uma estratégia, f) como se desdobra objetivo geral em objetivos específicos e g) como se relaciona objetivos com suas respectivas estratégias.

\begin{tabular}{|l|l|l|}
\hline Assunto & Conhecimentos & Habilidades \\
\hline Planejamento & O que é & Como é feito \\
\hline Objetivo & O que é & Como identificar \\
\hline Objetivo & O que deve ter & Como é redigido \\
\hline Objetivo & Tipos & Como desdobrar \\
\hline Estratégia & O que é & Como identificar \\
\hline Estratégia & O que deve ter & Como desenhar \\
\hline Estratégia & Relação com o objetivo & Como relacionar com os objetivos \\
\hline
\end{tabular}

Quadro 1. Quadro sintético de assuntos, conhecimentos e habilidades para avaliação.

Cabe notar, com base no Quadro 1, que os conhecimentos e as habilidades, nessa sistemática de avaliação, são como verso e reverso de um mesmo assunto ou perspectiva de assunto. Três assuntos, que representam os fatos e fenômenos do universo organizacional que os administradores devem dominar, foram avaliados (planejamento, objetivo e estratégia). Esse domínio se faz ora sob forma explicativo-analítica, ora sob aspectos práticos, instrumentais - muitas vezes, normativos e prescritivos. O que importa é que o avaliador tenha bem definido o que vai ser avaliado. É importante esclarecer, também, que essa clareza quanto à avaliação, quando repassada aos alunos, permite maior eficiência e eficácia nos estudos e na preparação para a avaliação.

\subsection{Terceira etapa: escolha do formato do teste}

A partir do Quadro 1 pode-se determinar o formato do teste. Nesse caso, foram identificados sete assuntos, com consequentes sete questões para avaliar os conhecimentos, e sete questões para avaliar as habilidades, totalizando 14 questões objetivas. Optou-se pelo seguinte sequenciamento: as questões de 1 a 7 mediram os conhecimentos, ao passo que as questões de 8 a 14 mediram as habilidades. Duas questões discursivas foram acrescentadas para medir o grau de correspondência escrita entre os conhecimentos e as habilidades sobre como se redige objetivos e estratégias e como se elabora planos.

\begin{tabular}{|l|l|c|l|c|}
\hline Assunto & Conhecimentos & Questões & Habilidades & Questões \\
\hline Planejamento & O que é & 1 & Como é feito & 8 \\
\hline Objetivo & O que é & 2 & Como identificar & 9 \\
\hline Objetivo & O que deve ter & 3 & Como é redigido & 10 \\
\hline Objetivo & Tipos & 4 & Como desdobrar & 11 \\
\hline Estratégia & O que é & 5 & Como identificar & 12 \\
\hline Estratégia & O que deve ter & 6 & Como desenhar & 13 \\
\hline Estratégia & Relação com o objetivo & 7 & Como relacionar com os objetivos & 14 \\
\hline
\end{tabular}

Quadro 2. Quadro sintético de assuntos, conhecimentos e habilidades para avaliação.

Partindo do Quadro 2, é possível perceber as correspondências, no formato do teste, entre as questões voltadas para medir conhecimentos e as voltadas para mensurar habilidades. Essa disposição vai ser fundamental para os processos de avaliação e replanejamento, a serem mostrados mais adiante no texto. A prática dessa sistemática levou à elaboração desses quadros esquematizadores para facilitar todas as atividades desta etapa em diante. 


\subsection{Quarta etapa: redação das questões}

A redação das questões foi feita na sequência do formato da prova constante no Quadro 2. A questão 1 foi redigida juntamente com a questão 8 , a questão 2 com a questão 9 , a questão $3 \mathrm{com}$ a 10 e, assim, sucessivamente, até o final das questões objetivas. As questões discursivas foram redigidas com base em um objetivo bem definido, além de medir conhecimentos e habilidades: dominar o formato sugerido por Nascimento-e-Silva (2012) para questões discursivas, que representa uma forma de organizar o raciocínio técnico-científico e colocá-lo de forma compreensiva em redações. Assim, toda redação que não estiver em conformidade com o formato definido foi considerada nula.

\subsection{Quinta etapa: definição das normas de resolução do teste}

As normas definidas foram as seguintes:

a) cada questão objetiva teria um tempo médio esperado para a resolução de três minutos. Como o teste era composto por 14 questões objetivas, o tempo total para as questões objetivas foi de 42 minutos;

b) cada questão discursiva teve de seguir o formato sugerido por Nascimento-e-Silva (2012), o qual dispõe que o texto deve começar respondendo a questão de forma sucinta, em forma afirmativa, seguida de um ponto em seguida; depois o aluno tem de completar o parágrafo explicando o que a afirmativa quer dizer e colocar outro ponto em seguida; por fim, a conclusão da redação deve apresentar um exemplo da explicação;

c) o mínimo e máximo de linhas para cada questão discursiva foi de 10 e 12, respectivamente, e o texto, a partir do formato definido, tinha de ser redigido em um único parágrafo;

d) o tempo médio para cada questão discursiva foi determinado em 10 minutos, com o tempo total de 20 minutos para o teste;

e) tempo total para a realização do teste foi definido em 62 minutos, sendo adicionados mais 8 minutos para que todos lessem as normas e orientações da prova, constantes na primeira página do teste, perfazendo um total de 70 minutos para a avaliação;

f) foi determinado que não poderia ser utilizado qualquer tipo de material que não fosse uma caneta esferográfica de tinta azul, que respostas rasuradas, a lápis, com dupla marcação ou incompreensíveis seriam consideradas erradas e que nenhuma forma de esclarecimento poderia ser fornecida porque a interpretação das questões também estaria sendo avaliada.

\subsection{Sexta etapa: pré-teste da avaliação}

Uma semana antes do teste foi feito o pré-teste. Esta etapa foi feita com uma simulação do teste contendo quatro questões objetivas e uma discursiva. Cada aluno calculou seu tempo para a resolução da questão e todos resolveram as questões com menos de três minutos para as questões objetivas e menos de 10 minutos para a discursiva. Esclarecimentos e outras simulações foram feitas, para que todos se familiarizassem com o tipo de avaliação. O quadro sintético da avaliação foi feito à vista de todos e serviu como fator motivador para a turma realizar o teste na semana seguinte. 


\subsection{Sétima etapa: realização do teste}

O teste foi aplicado normalmente. No entanto, vale salientar que a turma ficou "em clima de concurso público", no qual a competição havia ficado bastante acirrada, diferentemente das outras experiências de avaliação. Muitos relataram, posteriormente, que passaram a semana toda esperando o dia do teste para, pela primeira vez, saber o quanto tinham progredido no aprendizado de sua futura profissão.

\subsection{Oitava etapa: organização dos dados}

Os dados foram organizados com a ajuda da planilha eletrônica do Excel, como mostra a Figura 2. As colunas representam os alunos com seus nomes reais omitidos, $\mathrm{O} 1$ a $\mathrm{O} 14$ representam as questões objetivas do teste, D1 e D2 as duas questões discursivas. "Nota" é o resultado da mensuração quanto aos acertos nas questões objetivas e discursivas, NO representa a nota obtida nas questões objetivas e ND é a nota obtida nas questões discursivas.

As linhas de A1 até $A 29$ representam os alunos e seus respectivos escores -. isso é o que se chama, tecnicamente, mensuração na implementação de sistemas de controle. Cabe notar que, nessa sistemática, o que está sendo controlado é a aprendizagem dos alunos em relação a planejamento, objetivo e estratégias. Esses números representam o final da segunda etapa do controle, que é a mensuração.

Abaixo da planilha, "acertos" representa a quantidade de acertos dos alunos para cada questão. Essa sistemática avalia também o desempenho da turma, o que permite comparar desempenho de turmas semelhantes. "Acertos \%" é a mesma informação de desempenho da turma, porém, medida em termos percentuais, o que indica maior sintetização de resultados do que a frequência simples. Os três números contínuos que aparecem nas casas da linha "acertos" com as colunas "nota", NO e ND são as médias desses indicadores, ou seja, a média referente às notas (média global), às questões objetivas e às questões discursivas. Finalmente, a última linha da planilha apresenta os desvios padrões da nota global, nota das questões objetivas e nota das questões discursivas.

\subsection{Nona etapa: avaliação dos resultados}

Com base nos resultados apresentados na Figura 2, é possível chegar às seguintes conclusões em relação à demonstração da avaliação da aprendizagem da turma avaliada:

a) Os alunos $A 3, A 7, A 9, A 12, A 17, A 20$ e $A 29$ não obtiveram sucesso na avaliação global da aprendizagem, que exigia nota global mínima igual a 6,0, o que os coloca imediatamente no grupo de recuperação e aprendizagem, objeto da décima etapa dessa sistemática. Esses alunos terão que recuperar os conteúdos assinalados com $X$ na planilha.

b) Os alunos A7, A17 e A29 também terão que recuperar a capacidade de expressar resolução de problemas escritos, uma vez que obtiveram nota zero nas questões discursivas. A análise dos testes desses alunos indicou que o fracasso foi decorrente de não terem dominado o formato lógico da redação, uma vez que dominam os conteúdos avaliados. Por sua vez, os alunos A22 e A26, apesar de terem obtido nota global igual ou superior a 6,0, não obtiveram o desempenho necessário nas questões discursivas, o que exige deles recuperação dessa habilidade. 


\begin{tabular}{|c|c|c|c|c|c|c|c|c|c|c|c|c|c|c|c|c|c|c|c|c|}
\hline A & B & C & D & $E$ & $\mathrm{~F}$ & G & H & 1 & J & K & L & M & $\mathrm{N}$ & 0 & P & Q & R & $\mathrm{s}$ & $\mathrm{T}$ & U \\
\hline ALUNO & 01 & 02 & 03 & 04 & 05 & 06 & 07 & 08 & 09 & 010 & 011 & 012 & 013 & 014 & D1 & D2 & NOTA & NO & ND & \\
\hline A1 & 0,5 & $\mathrm{x}$ & 0,5 & 0,5 & $\mathrm{x}$ & 0,5 & 0,5 & $x$ & $\mathrm{x}$ & 0,5 & 0,5 & 0,5 & 0,5 & 0,5 & $\mathrm{x}$ & 1,5 & 6,5 & 5,0 & 1,5 & \\
\hline A2 & 0,5 & 0,5 & 0,5 & $x$ & 0,5 & 0,5 & 0,5 & 0,5 & 0,5 & 0,5 & $\mathrm{x}$ & 0,5 & 0,5 & 0,5 & 1,5 & 1,5 & 9,0 & 6,0 & 3,0 & \\
\hline A3 & 0,5 & $x$ & $x$ & $x$ & 0,5 & 0,5 & 0,5 & $x$ & $x$ & $x$ & $x$ & 0,5 & 0,5 & 0,5 & 1,5 & $x$ & 5,0 & 3,5 & 1,5 & \\
\hline A4 & 0,5 & 0,5 & 0,5 & 0,5 & 0,5 & 0,5 & 0,5 & 0,5 & 0,5 & 0,5 & 0,5 & 0,5 & 0,5 & 0,5 & 1,5 & 1,5 & 10,0 & 7,0 & 3,0 & \\
\hline A5 & $x$ & 0,5 & 0,5 & 0,5 & 0,5 & 0,5 & 0,5 & 0,5 & $x$ & 0,5 & 0,5 & 0,5 & 0,5 & 0,5 & $x$ & 1,5 & 7,5 & 6,0 & 1,5 & \\
\hline A6 & 0,5 & 0,5 & 0,5 & 0,5 & 0,5 & 0,5 & 0,5 & 0,5 & $x$ & 0,5 & 0,5 & 0,5 & 0,5 & 0,5 & 1,5 & $\mathrm{x}$ & 8,0 & 6,5 & 1,5 & \\
\hline A7 & 0,5 & $\mathrm{x}$ & $\mathrm{x}$ & $x$ & 0,5 & 0,5 & 0,5 & 0,5 & $x$ & $x$ & $\mathrm{x}$ & 0,5 & 0,5 & 0,5 & $x$ & $\mathrm{x}$ & 4,0 & 4,0 & 0,0 & \\
\hline A8 & 0,5 & 0,5 & $\mathrm{x}$ & 0,5 & $x$ & 0,5 & $x$ & 0,5 & 0,5 & $x$ & 0,5 & 0,5 & 0,5 & 0,5 & 1,5 & 1,5 & 8,0 & 5,0 & 3,0 & \\
\hline A9 & $\mathrm{x}$ & 0,5 & 0,5 & 0,5 & $\mathrm{x}$ & 0,5 & 0,5 & 0,5 & $x$ & $x$ & $\mathrm{x}$ & 0,5 & 0,5 & $x$ & 1,5 & $x$ & 5,5 & 4,0 & 1,5 & \\
\hline A10 & 0,5 & $\mathrm{x}$ & 0,5 & $x$ & 0,5 & $\mathrm{x}$ & 0,5 & 0,5 & 0,5 & 0,5 & $\mathrm{x}$ & 0,5 & 0,5 & 0,5 & 1,5 & 1,5 & 8,0 & 5,0 & 3,0 & \\
\hline A11 & 0,5 & 0,5 & $\mathrm{x}$ & 0,5 & 0,5 & 0,5 & $\mathrm{x}$ & 0,5 & 0,5 & 0,5 & 0,5 & 0,5 & 0,5 & 0,5 & $x$ & 1,5 & 7,5 & 6,0 & 1,5 & \\
\hline A12 & 0,5 & 0,5 & $\mathrm{x}$ & 0,5 & $x$ & $\mathrm{x}$ & 0,5 & 0,5 & $x$ & $x$ & $x$ & $x$ & $x$ & 0,5 & 1,5 & $x$ & 4,5 & 3,0 & 1,5 & \\
\hline A13 & $\mathrm{x}$ & 0,5 & 0,5 & 0,5 & $\mathrm{x}$ & 0,5 & 0,5 & 0,5 & $\mathrm{x}$ & 0,5 & 0,5 & 0,5 & 0,5 & 0,5 & 1,5 & 1,5 & 8,5 & 5,5 & 3,0 & \\
\hline A14 & 0,5 & 0,5 & $x$ & 0,5 & 0,5 & 0,5 & $x$ & 0,5 & $\mathrm{x}$ & 0,5 & 0,5 & 0,5 & 0,5 & 0,5 & $\mathrm{x}$ & 1,5 & 7,0 & 5,5 & 1,5 & \\
\hline A15 & 0,5 & 0,5 & $x$ & 0,5 & 0,5 & 0,5 & 0,5 & 0,5 & $x$ & 0,5 & 0,5 & 0,5 & 0,5 & 0,5 & 1,5 & $x$ & 7,5 & 6,0 & 1,5 & \\
\hline A16 & 0,5 & 0,5 & $x$ & 0,5 & 0,5 & 0,5 & 0,5 & 0,5 & 0,5 & $x$ & 0,5 & 0,5 & 0,5 & 0,5 & 1,5 & 1,5 & 9,0 & 6,0 & 3,0 & \\
\hline A17 & $x$ & 0,5 & $x$ & 0,5 & $x$ & 0,5 & 0,5 & 0,5 & $x$ & $x$ & $x$ & 0,5 & 0,5 & $x$ & $x$ & $x$ & 3,5 & 3,5 & 0,0 & \\
\hline A18 & 0,5 & 0,5 & $x$ & 0,5 & 0,5 & 0,5 & $x$ & 0,5 & 0,5 & 0,5 & 0,5 & 0,5 & 0,5 & 0,5 & 1,5 & 1,5 & 9,0 & 6,0 & 3,0 & \\
\hline A19 & 0,5 & 0,5 & 0,5 & 0,5 & 0,5 & 0,5 & $x$ & 0,5 & $x$ & $x$ & 0,5 & 0,5 & 0,5 & 0,5 & 1,5 & $x$ & 7,0 & 5,5 & 1,5 & \\
\hline A20 & $x$ & $x$ & $x$ & 0,5 & 0,5 & 0,5 & 0,5 & $x$ & $x$ & $x$ & 0,5 & $x$ & 0,5 & 0,5 & 1,5 & $x$ & 5,0 & 3,5 & 1,5 & \\
\hline A21 & 0,5 & 0,5 & 0,5 & 0,5 & $x$ & 0,5 & $\mathrm{x}$ & 0,5 & 0,5 & 0,5 & 0,5 & 0,5 & 0,5 & 0,5 & 1,5 & 1,5 & 9,0 & 6,0 & 3,0 & \\
\hline A22 & 0,5 & 0,5 & $x$ & 0,5 & 0,5 & 0,5 & 0,5 & 0,5 & 0,5 & 0,5 & 0,5 & 0,5 & 0,5 & 0,5 & $x$ & $x$ & 6,5 & 6,5 & 0,0 & \\
\hline A23 & 0,5 & 0,5 & $x$ & 0,5 & $x$ & 0,5 & 0,5 & 0,5 & $x$ & 0,5 & 0,5 & $x$ & 0,5 & 0,5 & 1,5 & $x$ & 6,5 & 5,0 & 1,5 & \\
\hline A24 & 0,5 & 0,5 & 0,5 & 0,5 & 0,5 & 0,5 & 0,5 & 0,5 & 0,5 & 0,5 & $x$ & 0,5 & $x$ & 0,5 & 1,5 & $x$ & 7,5 & 6,0 & 1,5 & \\
\hline A25 & 0,5 & 0,5 & $x$ & 0,5 & 0,5 & 0,5 & 0,5 & 0,5 & $x$ & 0,5 & 0,5 & 0,5 & 0,5 & 0,5 & 1,5 & 1,5 & 9,0 & 6,0 & 3,0 & \\
\hline A26 & 0,5 & 0,5 & 0,5 & 0,5 & $x$ & 0,5 & 0,5 & $x$ & 0,5 & 0,5 & 0,5 & 0,5 & 0,5 & 0,5 & $x$ & $x$ & 6,0 & 6,0 & 0,0 & \\
\hline A27 & 0,5 & $x$ & 0,5 & 0,5 & 0,5 & 0,5 & 0,5 & 0,5 & $x$ & 0,5 & 0,5 & 0,5 & 0,5 & 0,5 & 1,5 & 1,5 & 9,0 & 6,0 & 3,0 & \\
\hline A28 & 0,5 & 0,5 & 0,5 & 0,5 & 0,5 & 0,5 & 0,5 & 0,5 & 0,5 & 0,5 & 0,5 & 0,5 & $x$ & 0,5 & 1,5 & $x$ & 8,0 & 6,5 & 1,5 & \\
\hline A29 & 0,5 & $x$ & $x$ & $x$ & 0,5 & 0,5 & 0,5 & $x$ & $x$ & 0,5 & 0,5 & 0,5 & $x$ & 0,5 & $x$ & $x$ & 4,0 & 4,0 & 0,0 & \\
\hline ACERTOS & 24 & 22 & 14 & 24 & 20 & 27 & 23 & 24 & 12 & 20 & 21 & 26 & 25 & 27 & 20 & 14 & 7,3 & 5,5 & 1,8 & \\
\hline ACERTOS \% & 80 & 73 & 47 & 80 & 67 & 90 & 77 & 80 & 40 & 67 & 70 & 87 & 83 & 90 & 67 & 47 & & & & \\
\hline Desvio padrão & & & & & & & & & & & & & & & & & 1,7 & 1,07 & 1,05 & \\
\hline
\end{tabular}

Figura 2. Quadro sintético de avaliação de aprendizagem

c) O desempenho global da turma foi de 7,3, o que representa um resultado satisfatório, resultado da soma da média 5,5 referente às questões objetivas somado com a média 1,8 referente às discursivas. 0 desvio padrão de 1,7 para o desempenho global e de 1,07 e 1,05 para as questões objetivas e discursivas corrobora essa avaliação positiva da aprendizagem da turma.

d) Das 14 questões, apenas três foram consideradas insatisfatórias. Essas questões são a 03, 09 e D2. A questão 03, de acordo com o quadro 2, avaliou se os alunos sabiam quais são os elementos obrigatórios em um objetivo, a questão 09 procurou saber se estes são capazes de identificar um objetivo e a questão D2 solicitou aos alunos que descrevessem como os objetivos devem ser redigidos. Esses resultados demonstram que o assunto "objetivos" precisa ser reensinado. De fato, quando se procura saber quais foram as questões que levaram os alunos ao fracasso, percebe-se que este foi o assunto central.

\subsection{Décima etapa: análise das causas dos fracassos e a análise do replanejamento}

Apesar de o desempenho da turma poder ser considerado um sucesso, nove alunos obtiveram algum tipo de fracasso, seja por insucesso na avaliação global, seja por algum aspecto da avaliação. Os resultados indicaram duas causas: falta de conhecimento sobre o assunto "objetivos" e falta de habilidade para redigir questões discursivas. No entanto, é possível que outros fatores possam ter concorrido para gerar o fracasso desses alunos. Então, procedeu-se ao mapeamento das causas com o uso do diagrama de Ishikawa. 
Os alunos foram entrevistados em grupo em busca das possíveis causas dos seus desempenhos. O resultado da entrevista permitiu a elaboração do diagrama apresentando na Figura 3. Nota-se que a maior carga dos fatores que levaram ao insucesso dos alunos na avaliação está centrada na figura da matéria-prima, representada pelos próprios alunos, tais como problemas de leitura e interpretação de texto; falta de concentração, principalmente porque a aula é realizada logo após o almoço; o fato de não terem consultado o material disponível no sistema da instituição e, finalmente, porque não estudaram o mínimo que eles próprios consideravam razoável para tal.

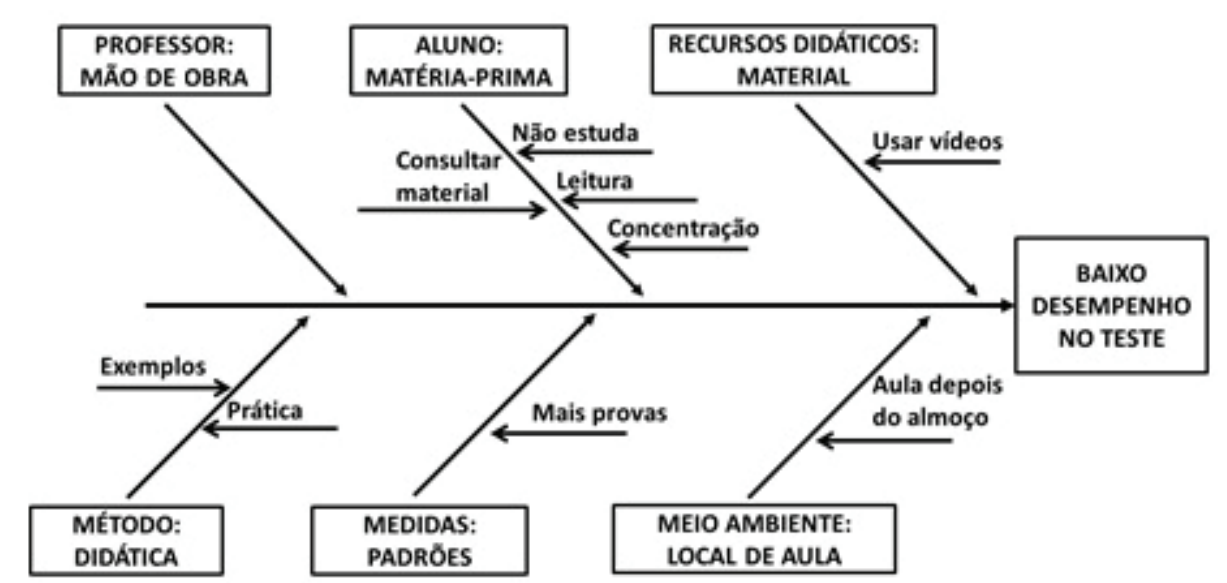

Figura 3. Causas do fracasso na avaliação de desempenho.

Para efeito de replanejamento da aprendizagem, foi solicitado que vídeos fossem utilizados para dar visibilidade ao assunto "objetivos" e que nas aulas exemplos e situações práticas fossem mais explorados. Com relação ao fato de a aula ser realizada depois do almoço, foi combinada a realização de dez minutos de ginástica laboral "para acordar", além da realização de mais provas, ficando a cargo dos próprios alunos procurar provas de concursos e resolvê-las. Caso haja dúvidas em alguma questão, os alunos procurarão o professor.

O planejamento da recuperação foi feito com objetivos centrados no assunto-causa do fracasso, e executado ao longo de duas semanas. Os resultados mostraram a eficácia da sistemática, uma vez que todos os alunos conseguiram posicionar-se com melhores escores, em nova reavaliação relativa a todos os assuntos (planejamento, objetivos e estratégias). A média dos nove alunos foi de 7,6, com menor escore no valor de 6,5 e maior no de 8,5. Esse procedimento, muitas vezes realizado em inúmeras turmas de graduação, pós-graduação e cursos técnicos nas áreas de administração e engenharia de produção, tem mostrado-se extremamente eficiente para avaliar a aprendizagem humana.

\subsection{Discussão dos resultados}

A administração é a área do conhecimento humano que representa com maior efetividade a capacidade de transformar esquemas abstratos em realidade concreta. É por isso que o processo gerencial começa justamente na construção esquemática da abstração, de ordem cognitiva, e culmina com a finalização da execução da última etapa do projeto que concretiza o que a cabeça humana idealizou de forma esquemático-abstrata, como parece ser a relação performance x avaliação do estudo de Guerra-López e Toker (2011) e a de Bisbe e Malagueño (2012), ambos 
considerando a dinâmica ambiental. Dito de outra forma, a capacidade humana é tamanha que realiza proezas como colocar o homem na lua (ou em outro planeta) ou construir grandes obras, sejam estes as pirâmides do Egito ou hidrelétricas majestosas, como a de Itaipu.

Esse arsenal de conhecimentos está disponível para realizar feitos supostamente sin-

gelos, como é o caso da aprendizagem. É estranho para os profissionais da administração o fato de a aprendizagem não ter o mesmo tratamento técnico especializado que tem a produção de qualquer produto ou serviço. Ao longo de processo de produção de bens, inúmeras etapas de avaliação da qualidade do produto em elaboração são executadas, de maneira que o produto final, quando da inspeção da qualidade final, esteja em conformidade com as exigências do usuário/cliente final, como o estudo de Amit e Belcourt (1999) indica. Por que isso não acontece com a formação profissional, uma vez que o egresso das instituições de ensino é o produto final de seus sistemas de formação?

Nesse estudo, demonstrou-se a aplicação do processo gerencial na gestão da aprendizagem e, mais do que isso, detalhou-se como a avaliação da aprendizagem pode ser feita em conformidade com as técnicas da função controle. Isso parece ser suficiente para ir além, empregando mais do que apenas os conhecimentos da Pedagogia para a avaliação de aprendizagem, e mostrar que a formação de profissionais de administração pode ser organizada com base nos mesmos princípios utilizados para a produção de bens e de serviços. Assim, ao invés de arena exclusiva dos profissionais de educação, a gestão da aprendizagem e da educação parece estar mais intrinsecamente relacionada à Administração e Engenharia de Produção do que com qualquer outra que não essas.

Algumas questões podem esclarecer essa constatação. Por exemplo, quais técnicas de desenho de estratégias de produção são estudas em corpos teóricos consolidados além de Administração e Engenharia de Produção? Que profissionais podem utilizar cálculos complexos e sistemas de programação avançados que não os de gestão e produção para organizar o processo de formação profissional aliado a eficientes sistemas de padronização, mensuração, avaliação e retificações, que não os dessas duas áreas? A simplicidade e efetividade da sistemática aqui apresentada demonstram o quão completo pode ser o profissional de administração, caso a responsabilidade do gerenciamento seja transferida para os administradores, a exemplo do que é feito nos países considerados desenvolvidos.

Assim, o gerenciamento da educação e da avaliação do produto da educação não pode se ater exclusivamente a questões comportamentais ou filosóficas. Se essa questão tão extremamente importante da vida humana associada não se aproximar da preocupação que se tem para com a perfeição da produção de produtos e serviços (que, ainda assim, são imperfeitos), dificilmente os profissionais que as instituições de ensino superior colocam no mercado darão conta dos desafios de suas profissões. A probabilidade de os administradores conseguirem alcançar os objetivos das organizações, que são cada vez mais desafiadores, diminuirão, e a pretensão de construir uma sociedade mais homogênea e isonômica não passará de um sonho irrealizável. $\mathrm{E}$ a realização de sonhos é a especialidade dos administradores. Contudo, para isso, é necessário um sistema de formação consistente e sólido, realidade para a qual o processo gerencial e a avaliação da aprendizagem demonstrados aqui pretendem contribuir.

\section{CONCLUSÃO}

Este texto apresentou uma sistemática de avaliação da aprendizagem para a formação de administradores, partindo do princípio de que o processo gerencial pode ser aplicado na ges- 
tão da aprendizagem dos administradores como ferramenta eficiente e eficaz para a formação do administrador que a história presente e futura necessita. Se a aprendizagem for planejada, organizada, dirigida e controlada, em conformidade com as técnicas e os métodos gerenciais, um sistema efetivo de controle pode ser criado para garantir que o profissional detenha os conhecimentos, as habilidades e os comportamentos que a sociedade, usuária/cliente das instituições de ensino superior e demandante dos profissionais formados, requer.

Através de técnicas de organização da produção, a sistemática aqui apresentada torna-se efetiva no processo de aprendizagem de várias disciplinas de diferentes áreas e níveis de estudo em vários estados brasileiros. Esse sucesso incentivou a divulgação, em formato técnicocientífico, para apreciação e críticas de especialistas em gestão e engenharia, para que seja aperfeiçoada e possa contribuir para a concretização dos desejos dos indivíduos de ter uma profissão cujas competências incorporadas sejam do interesse da sociedade. Se os objetivos da formação estiverem de acordo com o desejado pela sociedade, a sistemática aqui apresentada auxiliará na garantia de que esses objetivos podem atingidos, beneficiando a sociedade quando da transformação do aluno bisonho no egresso profissional de Administração.

Apesar do rigor metodológico, este estudo apresenta duas limitações, mas que não comprometem os seus achados: primeira, está limitado à avaliação dos aspectos referentes aos conhecimentos e às habilidades do conjunto de competências, isto é, não avalia atitudes; e, segunda, avalia habilidades apenas no seu aspecto formal, deixando de lado a dimensão material, o que significa que a habilidade aqui é avaliada do ponto de vista cognitivo e não performativo.

Dessa forma, recomendam-se os seguintes estudos de aperfeiçoamento da sistemática: a) avaliar as competências (o conjunto de conhecimentos, habilidades e atitudes) na avaliação de aprendizagem, o que configuraria a aprendizagem total; b) ampliar as fontes de evidência da avaliação da formação profissional com observação do desempenho performativo em relação às competências incorporadas, especialmente as atitudes, não contempladas na sistemática apresentada; e c) comparar os resultados dessa sistemática com outros modelos de avaliação de aprendizagem.

\section{REFERÊNCIAS}

ALMEIDA, M. L.; PERES, A. M. Conhecimentos, habilidades e atitudes sobre a gestão dos formados de enfermagem de uma universidade pública brasileira. Investigación e Educación en Enfermería, v. 30, n. 1, p. 66-76, jan./abr. 2012.

AMIT, R.; BELCOURT, M. Human resources management processes: a value-creating source of competitive advantage. European Management Journal, v. 17, n. 2, p. 174-181, 1999.

AREND, R. J. Patterns of sustained performance: beginnings, paths and ends. European Management Journal, v. 28, n. 3, p. 236-249, jun. 2010.
BISBE, J.; MALAGUEÑO, R. Using strategic performance measurement systems for strategy formulation: does it work in dynamic environments? Management Accounting Research, 2012 (article in press).

BULLER, P. F.; McEVOY, G. M. Strategy, human resource management and performance: sharpening line of sight. Human Resource Management Review, v. 22, n. 1, p. 43-56, mar. 2012.

CAVUS, N. The evaluation of learning management systems using an artificial intelligence fuzzy logic algorithm. Advances in Engineering Software, v. 41, p. 248-254, 2010.

CAVUS, N.; MOMANI, A. M. Computer aided evaluation of learning management systems. Procedia Social and Behavioral Sciences, v. 1, p. 426-430, 2009. 
CHEN, H. L. An empirical examination of project contractor's supply-chain cash flow performance and owner's payment patterns. International Journal of Project Management, v. 29, n. 5, p. 604-614, jul. 2011.

CORDEIRO, R. A.; SILVA, A. B. Os estilos de aprendizagem influenciam $O$ desempenho acadêmico dos estudantes de finanças? Rev. Adm. UFSM, v. 5, n. 2, p. 243-261, mai./ago. 2012.

CUPPEN, A quasi-experimental evaluation of learning in a stakeholder dialogue on bioenergy. Research Policy, v. 41, p. 624-637, 2012.

GUERRA-LÓPEZ, I.; TOKER, S. An application of the impact evaluation process for designing a performance measurement and evaluation framework in K-12 environments. Evaluation and Program Planning, v. 35 , n. 2, p. 222-235, may. 2011.

JOHANSEN, W.; AGGERHOLM, H. K.; FRANDSEN, F. Entering new territory: a study of internal crisis management and crisis communication in organizations. Public Relations Review, v. 38, n. 2, p, 270-279, jun. 2012.

JOHNSON, D. S.; CLARK, B. H.; BARCZAK, G. Customer relationship management processes: how faithful are business-to-business firms to customer profitability? Industrial Marketing Management, 2012, doi:10.1016/j. indmarman.2012.04.001 (article in press).

KOC, M.; ERGINBAS, S. Does computersupported Math instruction makes students perceive them to be task-oriented and wellbehaved: an experimental study. Procedia Social and Behavioral Sciences, v. 15, p. 29272931, 2011.

LI, Y.; TAN, C.-H.; TEO, H.-H. Leadership characteristics and developer's motivation in open source software development. Information \& Management, 2012 (article in press).
LONDON, M.; SMITHER, J. W. Feedback orientation, feedback culture, and the longitudinal performance management process. Human Resource Management Review, v. 12, p. 81-100, 2002.

LULSEGGED, A. H. M. Teaching and management in education: can they be the same? International Journal of Educational Development, v. 4, n. 2, p. 113-121, 1984.

MAJOR, E.; ASCH, D.; CORDEY-HAYES, M. Foresight as core competence. Futures, v. 33, n. 2, p. 91-107, mar, 2001.

MARQUEZ, A. C.; GUPTA, J. N. D. Contemporary maintenance management: process, framework and supporting pillars. Omega, v. 34, p. 313-326, 2006.

MONTOYA-DEL-CORTE, J.; FARÍAS-MARTÍNEZ, G. M. Desarrollo de habilidades profesionales y adquisición de conocimientos en los programas académicos de administración de empresas y contaduría pública: una investigación exploratoria con estudiantes de España y México. Innovar, v. 21, n. 40, p. 93-106, may./ ago. 2011.

PETTS, N. Building growth on core competence: a practical approach. Long Range Planning, v. 30, n. 4, p. 551-561, aug. 1997.

ROBSON, L. S.; MACDONALD, S.; GRAY, G. C.; EERD, D. L. V.; BIGELOW, P. L. A descriptive study of the OHS management auditing methods used by public sector organizations conducting audits of workplaces: Implications for audit reliability and validity. Safety Science, v. 50, n. 2, p. 181-189, feb. 2012.

SCHWARTZ, A.; PFORR, F. The crisis communication preparedness of nonprofit organizations: the case of German interest groups. Public Relations Review, v. 37, n. 1, p. 68-70, mar. 2011.

SING, S. K.; WATSON, H. J.; WATSON, R. T. EIS support for the strategic management process. Decision Support Systems, v. 33, p. 71-85, 
2002.

SWEETSER, K. D.; KELLEHER, T.A survey of social media use, motivation and leadership among public relations practitioners. Public Relations Review, v. 37, n. 4, p. 425-428, nov. 2011.

USUNIER, J.-C. Language as a resource to assess cross-cultural equivalence in quantitative management research. Journal of World Business, v. 46, n. 3, p. 314-319, jul. 2011.

VARGAS, K. S.; BIRRER, J. A.; MINELLO, I. F. Estilos de aprendizagem e níveis de comprometimento organizacional: uma abordagem com residentes multiprofissionais da saúde. Rev. Adm. UFSM, Santa Maria, v. 5, n. 3, p. 589-606, set./dez. 2012.

WALTER, F.; BRUCH, H. Structural impacts on the occurrence and effectiveness of transformational leadership: an empirical study at the organizational level of analysis. The Leadership Quarterly, v. 21, n. 5, p. 765782, oct. 2010.

WRZESIEN, M.; RAYA, M. A. Learning in serious virtual worlds: evaluation of learning effectiveness and appeal to students in the E-Junior project. Computers \& Education, v. 55, p. 178-187, 2010.

WU, H.-Y.; LIN, Y.-K.; CHANG, C.-H. Performance evaluation of extension education centers in universities based on balanced scorecard. Evaluation and Program Planning, v. 34, n. 1, p. 37-50, feb. 2011. 